\title{
A Quantitative Evaluation Model of Interactive Whiteboard Classroom Based on K-Means Algorithm
}

\author{
https://doi.org/10.3991/ijet.v13i11.9603 \\ Feng-juan Liu \\ Shaanxi University of Technology, Shaanxi Hanzhong, China \\ fengjuanliu2287@126.com
}

\begin{abstract}
In order to apply the K-means algorithm to the quantitative evaluation model of interactive whiteboard class, the automatic correction method of the natural scene text plane image in the interactive whiteboard class is put forward. This method makes full use of the whiteboard border line information, and completes the whiteboard correction through the Canny operator edge detection, the PPHT straight line extraction, the K-means algorithm linear clustering, $\mathrm{H}$ matrix transformation and other steps. Finally, experiments are carried out to test the performance of the algorithm. The research results show that the algorithm has a high success rate of correction for basic simple background and simple content whiteboard, and the algorithm performance is relatively stable. It can be seen that the K-means algorithm has broad application prospects in the quantitative evaluation model of interactive whiteboard classroom.
\end{abstract}

Keywords - classroom evaluation, teaching interaction, evaluation model, data mining

\section{Introduction}

In the late last century, the blackboard chalk teaching has always been a necessary media tool in the current classroom teaching. The characteristics of the blackboard flexible writing can highlight its guiding role in the process of teaching content in the changeable classroom teaching environment, and also make the teachers' explanation and body language well cooperate and play. However, the limitations of board books are also relatively large, and the introduction of multimedia documents such as pictures, audio and audio in classroom teaching can only rely on other media to support the classroom teaching activities.

Because of the damage brought by dust to teachers and students, writing whiteboards gradually appeared. The writing whiteboard has not been widely used in the basic education environment because it needs the erasers of the whiteboard, the imprint on the whiteboard is difficult to eliminate, and the writing pen is easier to volatilize. Whiteboard usually refers to the writing plane made of white metal plate material. The function of the whiteboard is similar to that of the blackboard. It is a writing tool that can be repeatedly erased, usually used for teaching, meeting discussion, or personal and family notes. The whiteboard must be written with a special whiteboard pen. However, 
in recent years, due to the development of technology, whiteboard plane materials are also more diverse, not limited to white metal materials, but including glass plates, wood plates, PET materials, and ceramic plates. The color of the whiteboard itself is also developed from a single white color to white and a variety of color coexistence, and applied in a variety of scenes. On the other hand, with the development of information technology, interactive electronic whiteboard has also developed greatly. With the greater and greater role of whiteboard, the function is becoming stronger and stronger. The understanding and analysis of whiteboard plane image has attracted more and more attention, and the processing of whiteboard image is becoming more and more important.

The meeting has always been one of the means of communication and discussion. The meeting records are the witness of the whole meeting and the main way to get the contents of the meeting. The current meeting's written records are recorded by participants' handwriting and typing. There are obvious shortcomings in this way: when people attend a meeting, the most important thing is ideological communication, which makes the attention of the participants focus on the record itself and neglects the thinking of the current record, which is particularly common in the academic meeting. In addition, due to the restrictions on the number of meetings, many people who are unable to attend the meeting want to know the contents of the meeting. Traditional records also cause great obstacles to the sharing of records. The whiteboard is indispensable in many meetings, such as academic discussion, project planning, and patent declaration. People use whiteboard to write their ideas and express their views. It provides a very important sharing space for the participants.

With the development of IT industry, digital cameras have been widely embedded in mobile terminals, personal digital assistant (PDA) or notebook computers. People can get the image information of nature more conveniently. In the meeting, people can get whiteboard images conveniently through mobile phones, PDA, pocket computers and other devices, and use programs to process them. However, during the meeting, the relative position of the participants and the whiteboard is difficult to fix. The pictures taken are often contained in the three-dimensional perspective deformation, which greatly affects the performance of the computer program for whiteboard image processing. Therefore, it has great use value and research significance for the three-dimensional correction of whiteboard images. It can be seen that the development of electronic whiteboard to interactive whiteboard is a key point for the development of whiteboard technology. Interactive whiteboard and computer realize two-way transmission of data.

The principle of single image 3D correction is introduced, and a single projection transformation matrix is given by interactive method to get the mono - enantiomer transformation matrix from the word plane to the vertical plane of the camera. Then, the whiteboard class quantization is evaluated by the automatic correction algorithm of whiteboard image. The natural scene whiteboard image automatic correction algorithm based on K-means clustering algorithm is used to achieve the evaluation. 


\section{$2 \quad$ Literature Review}

As for the application and development of the interactive whiteboard in teaching, Britain, Canada, the United States, Australia and other countries started relatively early. In Britain, the interactive whiteboard got the attention and popularization of the government education institutions. By 2004, Britain was the first country to apply the interactive whiteboard in the world of education, and it was very quickly popularized and promoted in primary and secondary schools.

In the developed countries of Europe and America, there are many perspectives on the application of interactive whiteboard media in teaching. For example, Bested et al. (2017) discussed how to better use interactive whiteboard to teach and promote students' learning from the angle of changing teachers' teaching methods. In the classroom teaching, if teachers really want to use whiteboard for teaching, it is necessary to develop the whiteboard as the potential function of a mediator. Teachers need to adjust their teaching methods and design the functions of whiteboard according to the teaching objectives [1]. In order to reach this level, teachers have to learn how to adjust the interactive links in teaching design and teaching activities so that the interactive whiteboard provides effective support. If the interactive whiteboard cannot be applied to this level, only as a blackboard or playing video, animation, and picture, it will play a very small role in promoting the students' learning. Hillier et al. (2016) believed that, due to the use of interactive whiteboard in the classroom, students' enthusiasm for participation and response is very high, showing a strong learning motivation and learning interest [2]. Luo et al. (2016) did a research on the relationship between classroom whiteboard application and geometric achievement in the United States. The 53 teachers based on the interactive whiteboard teaching and 39 teachers who did not use interactive whiteboard are completed. The results showed that the difference between the two was great, the mean value of the former was 20.70 and the latter was only 11.48 [3].

The correction methods of natural scene text plane images can be roughly divided into two categories. One is automatic correction, the other is correction based on information provided by interactive methods.

According to the method of generating transformation matrix, the automatic correction method can be divided into two methods based on border and based on test selfcharacteristics. Each method has many limitations and can only be corrected for some specific types of natural scene text images.

The method of Onal et al. (2017) is a classic method to correct the characteristics of the text itself. In the method, firstly, the character, string and each text line are transformed into black regions, which are called black spots. Then, the nearest neighbor algorithm is used to calculate the nearest neighbors of each black spot, and the most significant linear combination is calculated by the greedy algorithm. The block spot set is used to extract horizontal direction information, that is, the position of horizontal vanishing point is obtained by the nearest clustering method [4]. Finally, the vertical direction information is extracted from the corrected image after achieving correction in horizontal direction. The vertical direction information extracted by this method is insufficient. Şengül and Türel (2017) used two main vanishing points to correct the textual images of perspective distortion. First of all, the parallel baselines in the text 
line and the blank information between the paragraphs were extracted, and then the positions of the horizontal vanishing point and the vertical vanishing point were estimated, respectively [5]. These two methods analyze the characters of the character itself in the text plane and calculate the single projection transformation matrix used for correction. However, these two algorithms have great limitations. If there are no long enough text lines in the text image or the text lines in the text image are too few, it is impossible to calculate the infinite point of the horizontal direction effectively, and the infinity of the vertical direction is more difficult to obtain.

Tunaboylu et al. (2016) proposed a method based on fuzzy logic and mathematical morphology to solve the three-dimensional perspective deformation of visual image [6]. This method first used mathematical morphological factors to extract characteristics of characters on text images, fitted out the text base line segments, and used fuzzy logic to calculate the vertical edges of characters, and then constructed a large number of quadrangular textual baselines. Finally, the calculated mono mapping transformation matrix was used to complete the correction of deformed image. Similarly, the algorithm cannot achieve good correction effect for text image with shorter text line, and the algorithm is based on the English text analysis. The huge difference in the structure of the Chinese characters and the English alphabet may make the morphological operator not effective. Therefore, the performance of the correction will be greatly influenced by the application of the algorithm to the analysis of Chinese characters. Umak et al. (2016) considered that the computer's operating interface was projected onto the interactive whiteboard through the projector, and the content of the computer could be operated and saved on the interactive whiteboard. The contents operated on the computer were displayed, operated and saved on the interactive whiteboard. With the support of the interactive whiteboard hardware and software, the interactive whiteboard provides a rich subject resource base and auxiliary teaching tool on the basis of the advantages of flexible blackboard writing and environmental pollution free [7].

To sum up, it can be seen that interactive whiteboard has been widely applied in recent years, and its popularity is very fast. However, according to the application level of teacher interaction whiteboard at present, through the literature and the questionnaire survey of some primary and secondary schools, it is necessary to make a profound analysis of the media characteristics, so as to fully and efficiently apply the media characteristics to the classroom teaching.

\section{Method}

\subsection{Homogeneous coordinate and single projection transformation}

First of all, the geometric relationship of the perspective projection imaging of the camera is analyzed. As shown in Figure 1, $\pi$ is the plane in which the point $\mathrm{p}$ actually lies in the space, and $\pi^{\prime}$ is the imaging plane of the camera. The $\mathrm{O}$ point in the figure is the optical center, and $\mathrm{OB}$ is the main axis of the camera. The Cartesian coordinate system composed of points $\mathrm{O}$ and $\mathrm{A}, \mathrm{B}$ and $\mathrm{C}$ axis is called the camera coordinate system. In order to describe any object in the space and the position of the image, the 
camera coordinate system can be used as the reference system. Any object in space is projected onto the plane $\pi^{\prime}$, which is perpendicular to the camera's optical axis. As shown in the graph, $p^{\prime}$ is the intersection point between $p$ and $O$ points on the plane $\pi^{\prime}$. All points in plane $\pi$ are also mapped to the $\pi^{\prime}$ plane through this way. Although the object in the three-dimensional space has been reduced to one dimension in the process of mapping to the imaging plane, the point in the space is not one-to-one correspondence with the points on the imaging plane. However, through Figure 1, it is seen that the points on the planes $\pi^{\prime}$ are one-to-one correspondence with the points on the plane $\pi$.

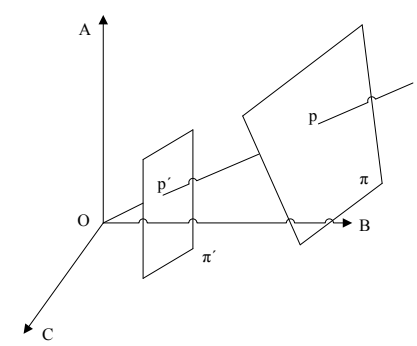

Fig. 1. The relationship among camera plane, image plane, and object plane

The concept of homogeneous coordinates is firstly introduced. A homogeneous coordinate is to represent an original $n$-dimensional vector by $n+1$ dimensional vector. That is to use three-dimensional vectors $(\mathrm{x}, \mathrm{y}, \mathrm{h})$ to represent two-dimensional vectors $(\mathrm{X}, \mathrm{Y})$. It can be seen from it that the homogeneous representation of a vector is not unique. The $\mathrm{k}$ value of the homogeneous coordinates takes the same point. For instance, the homogeneous coordinates $[8,4,2]$ and $[4,2,1]$ are two-dimensional point $[4,2]$. The function of homogeneous coordinates is that it provides an effective method to transform a set of points in two-dimensional, three-dimensional, or even high dimensional space by matrix operation, from one coordinate system to another. With homogeneous coordinate system, Formula (1) can be used to represent the coordinate transformation relation between points on two planes under perspective projection.

$$
\left[\begin{array}{c}
k x^{\prime} \\
k y^{\prime} \\
k
\end{array}\right]=\left[\begin{array}{lll}
h_{11} & h_{12} & h_{13} \\
h_{21} & h_{22} & h_{23} \\
h_{31} & h_{32} & h_{33}
\end{array}\right]\left[\begin{array}{c}
x \\
y \\
1
\end{array}\right] .
$$

$\mathrm{H}$ is used to represent the $3 * 3$ matrix in Formula (1), and $\mathrm{H}$ is also called the monography matrix (Homograph). For brevity, $\mathrm{m}$ ' is used to represent the homogeneous coordinates $\left(\mathrm{kx}^{\prime}, \mathrm{ky}^{\prime}, \mathrm{k}\right)^{\mathrm{T}}$, and $\mathrm{m}$ is used to represent the homogeneous coordinates $(\mathrm{X}$, $\mathrm{Y}, 1)^{\mathrm{T}}$, so that the Formula (1) becomes $\mathrm{m}^{\prime}=\mathrm{Hm}$. The homograph $\mathrm{H}$ here can be decomposed, and the $\mathrm{H}$ matrix is decomposed into three matrices, S, A and P. then, Formula (1) becomes Formula (2).

$$
m^{\prime}=E A P m
$$


Here $\mathrm{S}$ is a two-dimensional similarity transformation, $\mathrm{A}$ is an affine transformation, and $\mathrm{P}$ is a projection transformation. The decomposed forms are shown as follows:

$$
\begin{gathered}
S=\left[\begin{array}{ccc}
s \cos \theta & s \sin \theta & t_{x} \\
-s \sin \theta & s \cos \theta & t_{y} \\
0 & 0 & 1
\end{array}\right] . \\
A=\left[\begin{array}{ccc}
1 / b & -a / b & 0 \\
0 & 1 & 0 \\
0 & 0 & 1
\end{array}\right] . \\
P=\left[\begin{array}{lll}
1 & 0 & 0 \\
0 & 1 & 0 \\
l_{x} & l_{y} & 1
\end{array}\right] .
\end{gathered}
$$

It can be seen that the $\mathrm{H}$ matrix has 9 variables, but taking into account that the homogeneous coordinate has a parameter $\mathrm{k}$ reducible, so the $\mathrm{H}$ matrix actually has 8 degrees of freedom. Through the above decomposition, the two-dimensional similarity transformation matrix $S$ has four variables. $t_{x}$ and $t_{y}$ represent the translation of coordinates on the two axis, $\theta$ represents the rotation angle, and $\mathrm{S}$ represents the overall scaling ratio. In affine transformation matrix $\mathrm{A}$, $\mathrm{a}$ and $\mathrm{b}$ can represent affine scaling ratios in different directions. In projection matrix $\mathrm{P}, \mathrm{l}_{\mathrm{x}}$ and $\mathrm{l}_{\mathrm{y}}$ parameters represent an infinitely long line in the plane, and the formula is as follows.

$$
l_{x} \cdot x+l_{y} \cdot y+h \cdot 1=0
$$

The point $(\mathrm{x}, \mathrm{y}, \mathrm{h})^{\mathrm{T}}$ in the formula can satisfy the Formula (6) only in the case of $\mathrm{h}=0$, which means that only the infinity can satisfy the Formula (6). According to the rule that two points determine a straight line, Figure 2 gives the calculation process of the infinity straight line. In the image plane, two pairs of non-parallel lines are taken, and the intersection point of each pair of parallel lines is an infinite point in the direction of the parallel line, which connects the two infinity points. The obtained straight line is the infinite line of the image plane. As shown in Figure 2, in projective geometry, there is the following formula:

$$
\begin{aligned}
& V_{1}=l_{1} \times l_{2} \\
& V_{2}=l_{3} \times l_{4} \\
& l_{\infty}=V_{1} \times V_{2}
\end{aligned}
$$




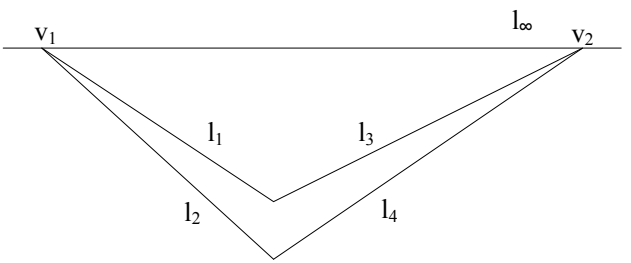

Fig. 2. Calculation of infinity line

\subsection{Interactive method for correcting text plane}

The grid itself contains the corresponding perspective transformation. The grid line corresponding to the horizontal direction of the rectangle box intersects the horizontal line of the rectangular box at the same infinite point, as shown in Figure 3. The grid lines perpendicular to the rectangular box intersects the vertical line of the rectangular box at the same infinite point. In fact, the grid is just like an equidistant ray crossing from two infinity points, the drawn grid is like the quadrilateral in a plane in a threedimensional space, and the display of the quadrangle is more stereoscopic.

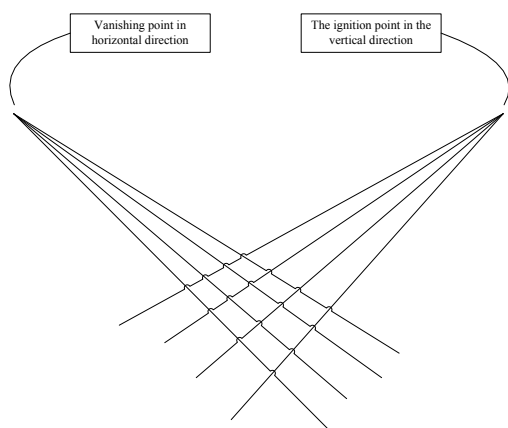

Fig. 3. Grid sketch map

It is necessary to point out that an important function of the program is that the quadrilateral frame can be stretched. The following border lines are stretched and it is ensured that the drawn border line is still intersected with the upper border line at the same infinite point. That is to say, the stretching frame is carried out on the principle of ensuring infinity invariance. This way ensures that the plane after stretching is still visually in the same three-dimensional plane with the original plane.

\section{$4 \quad$ Results and discussion}

\subsection{An overview of the automatic correction algorithm for whiteboard images}

The correction algorithm based on interactive method has the advantage of not being limited by image features in terms of application. However, for whiteboard images, if 
the features of the whiteboard image itself can be used, the algorithm will have better performance for processing the special natural scene text information images such as whiteboard images. Therefore, on this basis, an automatic correction algorithm for whiteboard image is proposed. The algorithm makes full use of the edge information of whiteboard image, extracts the edge information of whiteboard image, calculates the single projection correction matrix, and finally gets the corrected plane. The flow chart of the algorithm is shown in Figure 4.

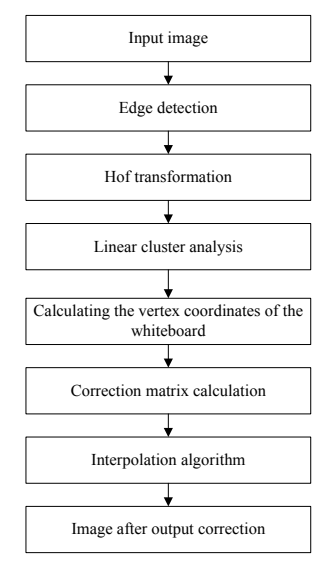

Fig. 4. Whiteboard correction algorithm flow chart

The algorithm first conducts gray-scale of the input image and carries out the edge detection. Then, the detected image is processed by Hough transform, and the four edges of the white board are extracted by clustering analysis of the lines detected by Hough transform. Moreover, four vertex coordinates of the whiteboard are calculated by four lines, and the four coordinates are used for the completion of whiteboard image correction.

\subsection{Edge detection of whiteboard image}

After the white board image is input, it is necessary to obtain the edge features of the whiteboard image effectively, so the edge operator is needed to extract the edge of the image. The edge detection is reflected by calculating the gray change rate of the domain. The commonly used edge extraction operators include Canny operator, Sobel operator, Prewitt operator and Laplasse Gauss (LoG) operator.

The Sobel operator uses the two convolution kernels in Figure 5 to extract the edges. These two kernels are applicable to horizontal and vertical boundaries, respectively. Each pixel in the image is convolution with the two convolution kernels, the maximum value of which is calculated as the output value of the point, and the result is the amplitude image of the boundary. 


\begin{tabular}{|c|c|c|}
\hline-1 & -2 & -1 \\
\hline 0 & 0 & 0 \\
\hline 1 & 2 & 1 \\
\hline
\end{tabular}

\begin{tabular}{|l|l|l|}
\hline-1 & 0 & 1 \\
\hline-1 & 0 & 2 \\
\hline-1 & 0 & 1 \\
\hline
\end{tabular}

Fig. 5. Sobel operator convolution sum

In order to reduce the influence of noise on image, smoothing filter is first processed by Gauss low-pass filtering before using Laplasse operator. The algorithm is shown in the following formula.

$$
\nabla^{2}[G(x, y) * f(x, y)]
$$

In the above formula, $\mathrm{G}(\mathrm{x}, \mathrm{y})$ represents the Gauss function. Using this convolution kernel, edges can be extracted from images. Similarly, the result of computation is the amplitude image of the boundary.

The Prewitt operator uses four operators to calculate each pixel of the image and get four gradient values. The same as the Sobel operator, the maximum gradient is chosen to replace the edge intensity, and the direction of the corresponding operator represents the edge direction. The four operators are shown in Figure 6.

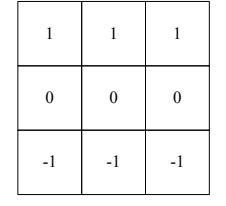

$0^{\circ}$ directional operator

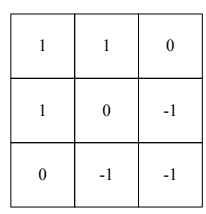

$45^{\circ}$ directional operator

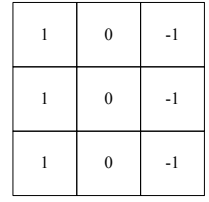

$90^{\circ}$ directional operator

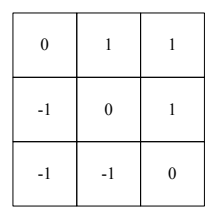

$135^{\circ}$ directional operator

Fig. 6. Prewitt operator schematic diagram

The Canny operator is a first-order operator, which was proposed by John Canny in 1986. The goal of Canny is to find an optimal edge detection algorithm. The optimal detection means that the algorithm can identify the actual edge of the image as much as possible, and the probability of missing the true edge and the probability of mis-checking the non-edge are as small as possible.

The optimal location criteria: the location of the detected edge points is nearest to the position of the actual edge point, or the minimum degree of the detected edge deviating from the real edge of the object due to the effect of noise. 
The detection point corresponds to the edge point: the edge points detected by the operator should be one-to-one correspondence with the actual edge points. To meet these requirements, Canny used the variational method, which is a way to find functions that satisfy specific roles. The optimal detection is expressed by four exponential function terms, but it is very similar to the first derivative of Gauss function.

Through the experiment, it is found that the edge noise is less and the edge is more complete with Canny operator processing, and the edge effect is better than that of other operators. From Figure 5, it can be seen that the Sobel operator and the Prewitt operator are very poor in detecting the left edge, and only some discrete points are detected, but the LoG operator and the Canny operator can detect a relatively complete line. It is proved that the Canny operator has higher accuracy than the gradient operator. In addition, the performance of the two operators is analyzed with the sensitivity of noise. It is pointed out that the LoG operator cannot eliminate the salt and pepper noise in the image and can easily calculate the double edge, while Canny is more sensitive to the high strength noise. Therefore, a more superior Canny algorithm is used to extract the edge of the whiteboard image.

\subsection{Line detection based on Hough transform}

After edge detection of whiteboard image, it is necessary to extract straight lines in the whiteboard image. Hough transform was first proposed by Paul Hough in 1962, and it implemented a mapping relationship from image space to parameter space. Hough transform maps complex edge features in image space into clustering detection in parameter space.

Hough can be used to process images after edge enhancement, which is a simple and effective method to extract edge features. It can extract lines, circles, ellipses, binary curves or even arbitrary shape edges. Hough transform has been widely concerned and widely applied in computer vision, military defense, office automation and other fields. The concrete idea is to transform the original image into the parameter space, to describe the line in the image by most boundary points meeting the parameter form, and to accumulate it by setting the accumulator to get the information needed for the point corresponding to the peak. Hough transform is highly valued by scholars in image processing, pattern recognition and computer vision because of its insensitivity to local defects, robustness to random noise and suitability for parallel processing. The outstanding advantage of Hough transform is that it can transform the difficult global detection problem into the local peak detection problem which is relatively easy to be solved in the parameter space. Moreover, the ability of Hough transform to detect geometry is also less disturbed by geometric breakpoints, and there is no need to preconnect or combine edge points. 


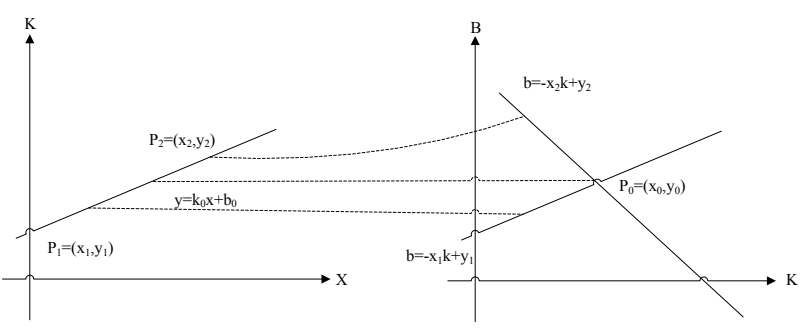

Fig. 7. The schematic diagram of Hough transformation in line detection

The principle of Hough transform is shown in Figure 7. The straight line $\mathrm{L}$ in the plane rectangular coordinate system on the left is expressed as:

$$
y=k x+b .
$$

In Formula (11), $\mathrm{k}$ is the slope, and $\mathrm{b}$ is the intercept. According to Formula (11), different points $(\mathrm{x}, \mathrm{y})$ on line $\mathrm{L}$ are transformed into a group of straight lines intersecting the point $\mathrm{P}$ in parameter space. Obviously, if the point $\mathrm{P}$ in the parameter space (local maximum) can be determined, the detection of straight line can be achieved.

Any straight line in the Cartesian coordinate system can also be expressed in polar coordinates, that is, it can be determined by two parameters of $\rho$ and $\alpha$, and for any point in the image space, its function relation is:

$$
\rho=x \cos \alpha+y \sin \alpha .
$$

$\rho$ is the distance between the origin and the straight line (the length of the vertical line of the origin to the straight line), and $\alpha$ determines the direction of the straight line (the angle between the vertical line of the origin to the straight line and the direction of the $\mathrm{x}$ axis). If the above transformation is carried out on the $\mathrm{n}$ point on the same line $\mathrm{L}$, the original image space $n$ points are corresponding to the $n$ bar sine curve in the parameter space, and the curves are intersected at the same point.

The strategy of Hough transform is to use the edge data points in the image space to calculate the possible locus of reference points in the parameter space, and count the calculated reference points in the accumulator, and finally select the peak value. This peak indicates that there is a line with more line points in the image space, and the parameters of the line are determined by the accumulator's $\rho$ and $\alpha$. In other words, it is determined according to $Q(i, j)=Q(i, j)+1$, and then the point $(x, y)$ in the image space meeting the above formula constitutes the straight line.

For whiteboard images, the whiteboard border in the image is detected by the Canny operator, and a clear border will be obtained. Relative to the writing content in the whiteboard, the straight line is relatively long. In view of this, the parameters of PPHT transformation are set. In this way, it is possible to better exclude the influence of whiteboard internal and whiteboard external short line on the performance of clustering analysis, so as to get better clustering results. The threshold of the shortest line length $\mathrm{T}$ the PPHT set is determined by Formula (13), in which width refers to the width of the 
whiteboard image read, height is the height of the whiteboard image read, and $\mathrm{n}$ is the setting parameter. According to the effect of actual correction, $\mathrm{n}$ takes 15 .

$$
T=(\text { width }+ \text { height }) / n
$$

The value of T should not be too small as it cannot play the role of screening straight lines. But it should not be too large, because through edge detection, due to the image quality and the influence of noise, the whiteboard image border cannot be connected together, which may form a few short lines. Canny edge detection is done here, and then Hough transform is used to get the final result. From the result, it can be seen that the number of straight lines in the images detected by PPHT with small $\mathrm{T}$ is quite large, and the average length is shorter. More short line interference can be seen in the images with larger $\mathrm{T}$ set.

\section{Conclusion}

Based on interactive 3D distortion correction for scene text images, the principle of three-dimensional correction of single image is introduced. A single projection transformation matrix of the text plane to the vertical plane of the camera through interactive method is given. Finally the three-dimensional correction is realized by the perspective transformation, and the recognition test and analysis of the Chinese characters in the corrected image are carried out. The acquisition of whiteboard image is similar to that of the natural scene image, both of which are through the camera. But the whiteboard image has its own characteristics, so the whiteboard can be seen as a special situation of the natural scene text plane. The K-means clustering algorithm is used to quantify the whiteboard image automatic correction algorithm of the natural scene to the whiteboard class.

\section{$6 \quad$ References}

[1] Bested, M., Weisberg, A., Durão, F. (2017). A social interactive whiteboard system us-ing finger-tracking for mobile devices: Multimedia Tools \& Applications, 76(1): 1-31.

[2] Hillier, E., Beauchamp, G., Whyte, S. (2016). A study of self-efficacy in the use of in-teractive whiteboards across educational settings: a european perspective from the itilt project: British Education Studies Association, 29(2): 49-79.

[3] Luo, Y., Shu, C. (2016). The effect of the interactive functions of whiteboards on ele-mentary students' learning: Journal of Educational Computing Research, 54(5): 78-82. https://doi.org/10.1177/0735633115628032

[4] Onal, N., Demir, C. (2017). The use of the interactive whiteboard in mathematics and mathematics lessons from the perspective of turkish middle school students: Interna-tional Journal of Higher Education, 6(3): 195.

[5] Şengül, M., Türel, Y. (2017). Teaching turkish as a foreign language with interactive whiteboards: a case study of multilingual learners: Technology Knowledge \& Learn-ing, 1(2): 115. https://doi.org/10.1007/s10758-017-9350-z 
Paper-A Quantitative Evaluation Model of Interactive Whiteboard Classroom Based on K-Means Algo...

[6] Tunaboylu, Ceren D., Ergül. (2016). The effect of teaching supported by interactive whiteboard on students' mathematical achievements in lower secondary education: Journal of Education \& Learning, 6(1): 81-94. https://doi.org/10.5539/jel.v6n1p81

[7] Umak, B., Punik, M., Heriko, M., Orgo, A. (2016). Differences between prospective, existing, and former users of interactive whiteboards on external factors affecting their adoption, usage and abandonment: Computers in Human Behavior, 72(C): 733-756.

\section{$7 \quad$ Author}

Feng-juan Liu is with Shaanxi University of Technology, Shaanxi Hanzhong,723000, China (fengjuanliu2287@126.com).

Article submitted 23 September 2018. Resubmitted 19 October 2018. Final acceptance 27 October 2019. Final version published as submitted by the author. 